\title{
Investigation on Present Bird Trouble of Electrical Power System and the Raise of a New Intelligent Environmental Bird-repellent Method
}

\author{
Xinyu Ma ${ }^{1, a}$ \\ ${ }^{1}$ North China Electric Power University, Beijing, 102206 \\ aalanncepu@foxmail.com
}

Keywords: Bird trouble; Pyroelectric infrared sensor; Solar panel; GPRS communication module

\begin{abstract}
Bird-trouble of Electrical power system has accounted for the third largest reason for transmission line fault. So it brings a huge influence on people's normal production and living. The paper researches and summarizes present bird-trouble of electrical power system and the existing methods of bird repellent measures. In the end, a new type of intelligent environmental bird repellent method is put forward.
\end{abstract}

\section{Introduction}

With the development of national economy, the supply of electricity has become the precondition of the development of each social field. As an indispensable part of normal supply of electric power, the transmission line itself is very fragile. Because most of the high voltage transmission lines distribute in the field, and ecological environment is changeable, they will be undoubtedly influenced by natural disasters and unpredictable damage of animals and so on. In the aspect of workload is a major challenge to the traditional artificial line-patrol inspection.

\section{The Cause for Bird-Trouble of Electrical Power System and Its Status Quo}

Compared with trees, transmission tower's structure is more stable, and it will not be easily shaken by rain and snow storm. So birds prefer to nest on the tower. Besides, there are few trees in some areas, so birds have to rest on the transmission lines and nest on them. If it rains, nests built with branches might be blown onto the charged wires or hanging bottles. Branches touch or stay close to the charged wire will cause short circuit grounding accident. Then, the whole lines will have the hidden trouble of tripping operation, which brings massive influence on people's production and normal life. Although some kinds of birds don't nest on transmission tower, they rest on the cross arm of the tower. Their excreta will pollute insulator chains. When the weather is humid or foggy, flashover accident will happen easily.

According To Information Statistics, after lightning stroke and external damage, bird-trouble is the third reason for transmission line malfunction.

\section{Present Bird Repellent Measures}

At present, there has been much traditional bird repellent measure on the market, including physical measures like hanging red flag and dead body of bird on the top, installing bell, retroreflector and windmills and so on. These measures are easy to achieve, but birds will not be afraid of them over time. They will not offer a long-term effect. There are also May chemical measures like spraying bird-repellent reagent. Although reagents are environmental-friendly, they have to be used almost every fifteen days. These measures are not universal and their effects are not obvious.

Recently, some new measures appear on the market. They chiefly use sound, light and heat to frighten or stimulate birds to repel birds. These products include radar bird-repellent device and ultrasonic wave bird-repellent device. These devices still exist some problems like hurting birds and inaccurate location. 


\section{The Raise of a New Intelligent Environmental Bird-Repellent Device}

The device is mainly composed of pyroelectric infrared sensor module, control module, voice module, communication module and power-supply module. It has advantages of safety, environmental-friendly, strong applicability, real-time monitoring, and low power consumption. The device is driven by passive pyroelectric infrared sensor (BISS0001) to realize a non-contact detection over long distance It is mainly used in electrical power systems to avoid some accidental interference such as bird-trouble.

When monitored objects enter the scope of infrared monitoring, infrared sensing device will be driven and output a weak electrical signal. After operation amplifying, filtering, power amplifying, the electrical signal is passed to the main control board. The device has semiconductor memory chips, and it can be connected to the computer via USB interface. Recordings can be downloaded through the data line and stored in the memory chip permanently. Sound can be played through audio power amplifier circuit and the use of external loudspeaker. On the other hand, main control board can pass the signal to the GPRS communication module and send warning messages to the security personnel.

1. Circuit Principle of Pyroelectric Infrared Sensor. Pyroelectric infrared sensor contains a pair of thermoelectric devices, a high value resistance and a low noise field effect transistor, which is installed in no.5 (the TO - 5) shell. Its structure and equivalent circuit are listed below.
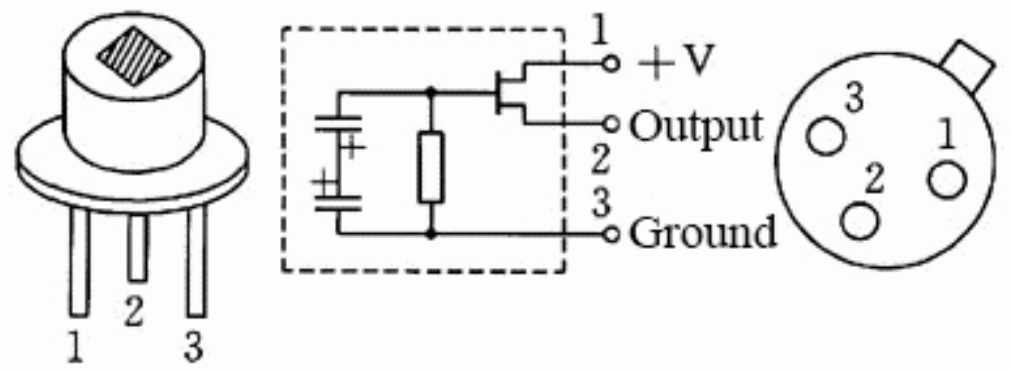

Figure 1.

Pyroelectric infrared sensor detects the infrared energy change of biological radiation with non-contact form. Pyroelectric infrared sensor sensitive unit T1, T2 change their temperature according to their feelings of infrared way. And the change of temperature leads to the change of the electrical signals. Because of the device's structure, temperature change of the environment and itself will not output signals. And the low frequency response of the sensor and the particular infrared wavelengths $(5 \sim 15$ microns $)$ response characteristic determines the senor only sensitive to the temperature changed by the outside infrared radiation. Put it another way, it is only sensitive to the movement of birds or related objects. The role of Fresnel lens is to make the output voltage signal in the form of pulse (the frequency of the pulse voltage is determined by the movement speed of the tested object, usually around $0.1 \sim 10$ hertz). In order to improve the detecting sensitivity of the detector to increase detection range, a Fresnel lens is usually installed in the front of the detector. Combined with the amplifying circuit, the signal can be amplified to $120 \sim 150 \mathrm{db}$, so that you the movements of birds in 10-meter range can be measured.

2. Principle of GPRS Communication Module. UB680 module integrates GSM/GPRS wireless communication module and GPS receiver module, GSM and GPS unifies highly. GPS information which is offered a variety of flexible working mode can be controlled and delivered locally and in the other places. 60 feet connector of board to board sets aside each function interface. Interfaces mainly include: GSM Power interfaces (Power Supply), GPS Power (Power Supply), the ADC interface, GSM serial communication interface (2 Channel UART), GPS serial communication interface (1 Channel UART), dual channel analog audio interface El (2 Channel Audio), 5x5 matrix keyboard interface (5x5 Keypad Array), standard SIM card interface, backup battery (RTC Backup), etc. The module itself embedded TCP/IP protocol, which supports the extension of AT command. 


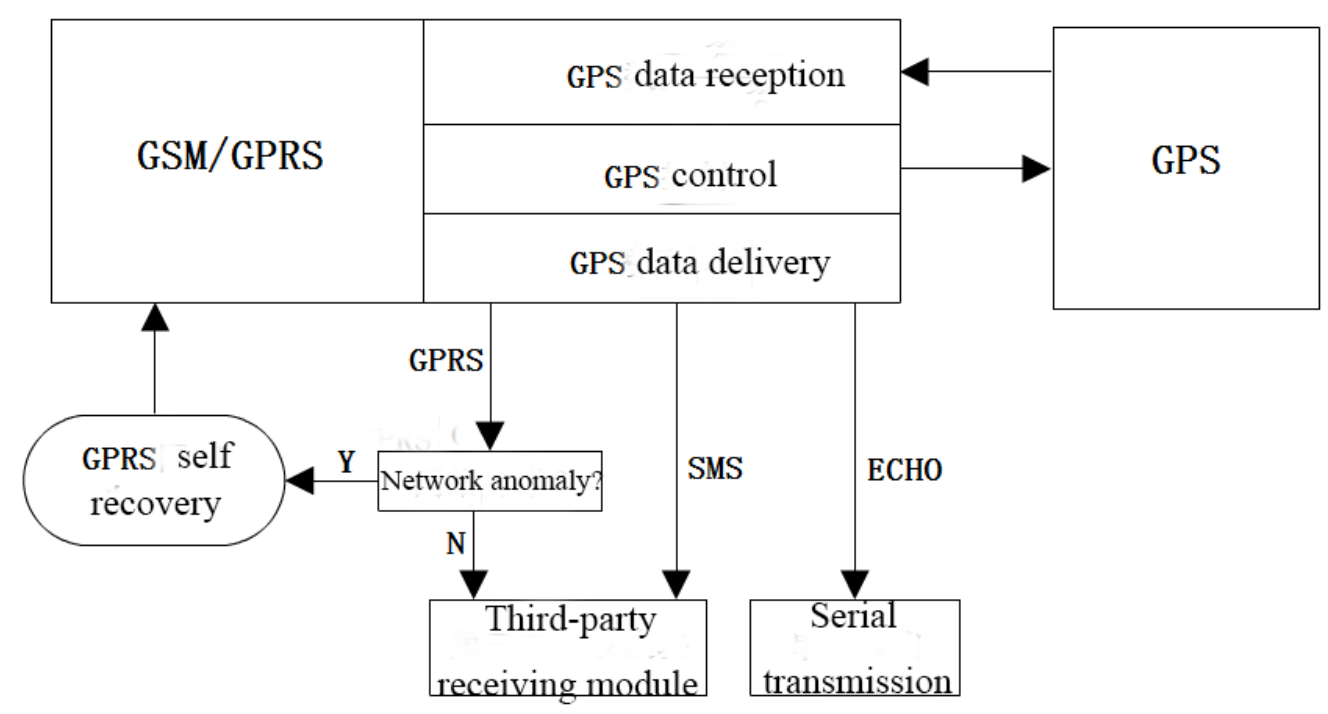

Figure 2.

3. Design of Signal Preprocessing Circuit. When the pyroelectric infrared sensor receives the infrared ray produced by the tested object, it will output a faint low-frequency signal to BISS0001 chip to preprocess it. At the same time, the DC potential is driven up to the VM. Then through internal bidirectional image detector, effective trigger signals VS will be detected to startup latency timer. When VDD $=5 \mathrm{~V}$, noise of $+/-1 \mathrm{~V}$ can be restrained effectively. It improves the reliability of the system.

COP3 is a comparator. When the input voltage $\mathrm{VC}<\mathrm{VR}(\mathrm{VR} \approx 0.2 \mathrm{VDD}), \mathrm{COP} 3$ outputs the low level, which blocks the AND gate U2 and bans trigger signal VS to deliver to the subordinate. When VC $>$ VR, COP3 outputs the high level, which opens the AND gate U2 and enters the delay period. At this moment, if there arrives any jump of trigger signal VS, the delay timer will be started, and the VO feet will output high level signal at the same time. Accessing the sound and light alarm circuit can realize the alarm of the signals

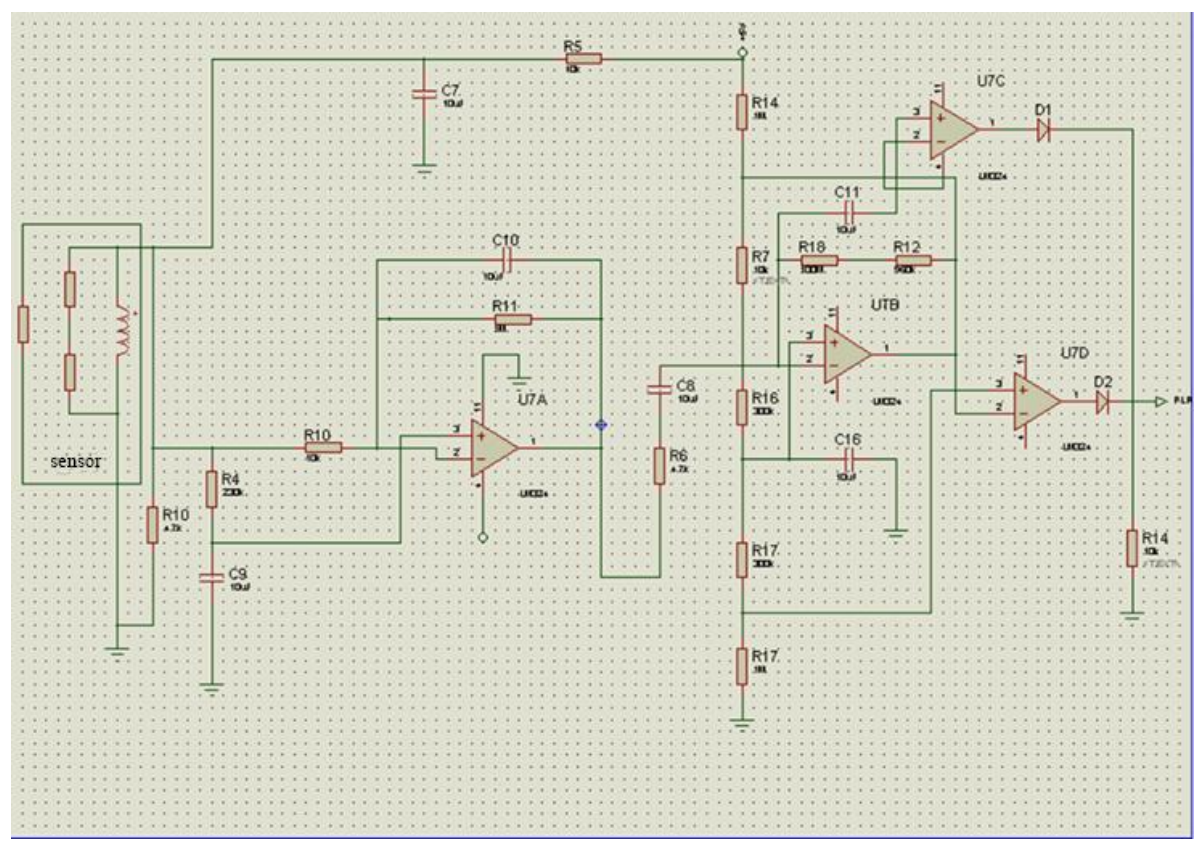

Figure 3. 
4. Design of Supply Circuit. Supply circuit can realize the switch between solar cell and accumulators. The circuit is shown in the figure below. Battery branch uses 2 diodes. When their voltages are equal, the device is still powered by solar panels. The method has automatic battery charging circuit. The solar modules convert solar energy into electricity and supply power to the load or recharge accumulators. Using a solar cell charging controller can protects the accumulators' charging and discharging. Accumulators are used to store electrical energy and transform direct current into alternating current. When it is sunny during the day, the solar cell will supply power to the equipment and charge the accumulators at the same time. When it is rainy during the day or at night, the solar cell components will not work. At this time, accumulators will take the charge of power supply.

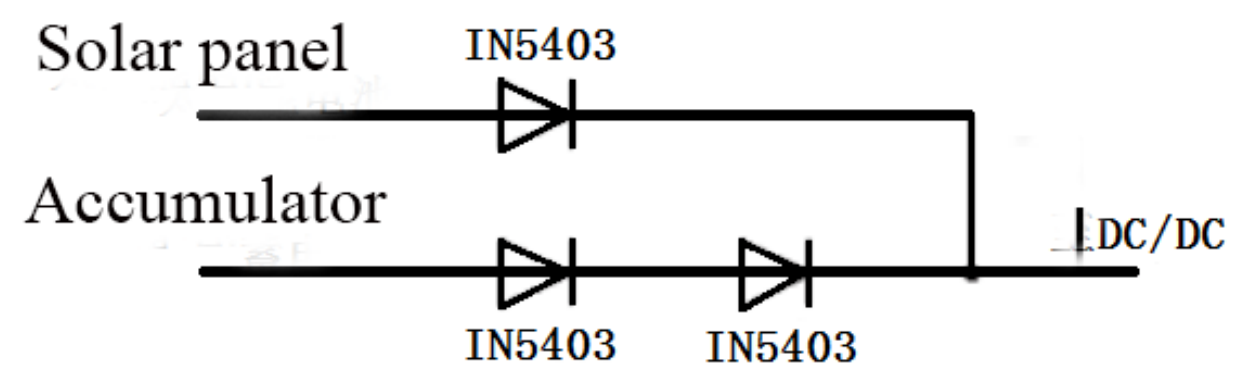

Figure 4.

\section{Conclusion}

Human and nature should live in harmony. We can't hurt birds while we should avoid the damage made by bird-trouble. So, we can only drive birds away instead of kill or hurt them. The paper concludes present bird-repellent devices and their disadvantages. On the basis of that, a new intelligent environmental bird-repellent device which can be used in electrical power system is raised. Besides, the way of making the device is also discussed. The device can not only drive birds away effectively, but also does no harm on them. It truly realizes ecological and green bird repellent and it protects ecological balance.

\section{References}

[1] Liu Peipei. Bird Damage investigation of fruit planting and fishery and the suggestions for the control methods[C]. Beijing Forestry University. 2011

[2] Zhao Wei, Lin Tong, Guo Jianquan, et al. Design of intelligent bio-acoustic frightening system against agriculture predators [J]. Transactions of the CSAE, 2010, 26(1):199-204.

[3] Harness R. E,K.R.Wilson.Electric-utility structures associated with raptor electrocutions in rural areas. Wildlife Society Bulletin. 2001.

[4] K. Bevanger, H. Brseth.Impact of power lines on bird mortality in a subalpine area. Animal Biodiversity and Conservation. 2004.

[5] Yang Minxiang. Research on Cause and Grade Division Method of Bird Damage in North China Power Grid[C]. North China Electric Power University. 2011.

[6] Chen Xiaozhi. Design and realization of warning model and system for transmission line anagianst bird damage[C]. University of Electronic science and Technology of China. 2014.

[7] Zhao Yuanyuan. Design and application of a bird driving device applied to electric power towers[C]. Dalian Maritime University. 2009. 
[8] P. Bayle. Preventing birds of prey problems at transmission lines in Western Europe. J. Raptor Res. 1999.

[9] Dirk Uwe Sauer,Jurgen Garche.Optimum battery design for applications in photovoltaic systems-the oretical considerations. Journal of Power Sources. 2001.

[10] Sundararajan, R.,Burnham, J.,Carlton, R.,Cherney, E.A.,Couret, G.,Eldridge, K.T.,Farzaneh, M.,Frazier, S.D.,Gorur, R.S.,Harness, R.,Shaffner, D.,Siegel, S.,Varner, J.Preventive measures to reduce bird-related power outages-Part I: Electrocution and collision. IEEE Transactions on Power Delivery. 2004. 